\title{
Dependency Detection in MobiMine and Random Matrices
}

\author{
Hillol Kargupta ${ }^{1}$, Krishnamoorthy Sivakumar ${ }^{2}$, and Samiran Ghosh ${ }^{1}$ \\ 1 Department of Computer Science and Electrical Engineering \\ University of Maryland Baltimore County \\ Baltimore, MD 21250, USA \\ $\{$ hillol, sghosh1\}@cs.umbc.edu \\ http://www.cs.umbc.edu/ hillol \\ 2 School of Electrical Engineering and Computer Science \\ Washington State University \\ Pullman, WA 99164-2752, USA \\ siva@eecs.wsu.edu
}

\begin{abstract}
This paper describes a novel approach to detect correlation from data streams in the context of MobiMine - an experimental mobile data mining system. It presents a brief description of the MobiMine and identifies the problem of detecting dependencies among stocks from incrementally observed financial data streams. This is a non-trivial problem since the stock-market data is inherently noisy and small incremental volumes of data makes the estimation process more vulnerable to noise. This paper presents EDS, a technique to estimate the correlation matrix from data streams by exploiting some properties of the distribution of eigenvalues for random matrices. It separates the "information" from the "noise" by comparing the eigen-spectrum generated from the observed data with that of random matrices. The comparison immediately leads to a decomposition of the covariance matrix into two matrices: one capturing the "noise" and the other capturing useful "information." The paper also presents experimental results using Nasdaq 100 stock data.
\end{abstract}

\section{Introduction}

Mobile computing devices like PDAs, cell-phones, wearables, and smart cards are playing an increasingly important role in our daily life. The emergence of powerful mobile devices with reasonable computing and storage capacity is ushering an era of advanced data and computationally intensive mobile applications. Monitoring and mining time-critical data streams in a ubiquitous fashion is one such possibility. Financial data monitoring, process control, regulation compliance, and security applications are some possible domains where such ubiquitous mining is very appealing.

This paper considers the problem of detecting dependencies among a set of features from financial data streams monitored by a distributed mobile data mining system called the MobiMine. MobiMine is not a market forecasting system.

T. Elomaa et al. (Eds.): PKDD, LNAI 2431, pp. 250-262, 2002.

(C) Springer-Verlag Berlin Heidelberg 2002 
It is neither a traditional system for stock selection and portfolio management. Instead it is designed for drawing the attention of the user to time critical "interesting" emerging characteristics in the stock market.

This paper explores a particular module of the MobiMine that tries to detect statistical dependencies among a set of stocks. At a given moment the system maintains a relevant amount of historical statistics and updates that based on the incoming block of data. Since the data block is usually noisy, the statistics collected from any given block should be carefully filtered and then presented to the user. This paper offers a technique for extracting useful information from individual data blocks in a data stream scenario based on some well-known results from the theory of randomized matrices. It presents a technique to extract significant Eigen-states from Data Streams (EDS) where the data blocks are noisy. The technique can also be easily applied to feature selection, feature construction, clustering, and regression from data streams. More generally, EDS offers a way to filter the observed data, so that any data mining technique (exploratory or otherwise) can later be applied on the filtered data. In the context of financial data streams, any technique for the analysing, forecasting, and monitoring stock prices can be applied on the filtered data. As such, the EDS by itself is not a market forecasting tool.

The technical approach of the proposed work is based on the observation that the distribution of eigenvalues of random matrices [1] exhibit some well known characteristics. The basic idea is to compare a "random" phenomenon with the behavior of the incoming data from the stream in the eigen space and note the differences. This results in a decomposition of the covariance matrix: one capturing the "noise" and the other capturing useful "information." Note that the terms "noise" and "information" are used in a generic sense. In the context of financial data, the change in price of a stock is influenced by two types of factors: (a) causal factors that directly or indirectly have an influence in the current or future performance of the company. This would include earnings, revenue, and future outlook of that company, performance of competitors, state of the overall economy, etc. This corresponds to the "information" part. (b) random factors that might be completely unpredictable and totally unrelated to the performance of the company. This corresponds to the "noise" part.

The eigenvectors generated from the "information" part of the covariance matrix are extracted and stored for the chosen application. Moreover, the eigenvectors can be used to filter the observed data by projecting them onto the subspace spanned by the eigenvectors corresponding to the "information."

Section 2 presents a brief overview of the MobiMine system. Section 3 discusses relevant theory of random matrices and then describes the EDS technique. Section 4 presents the experimental results. Section 5 concludes the work and identifies future work. 

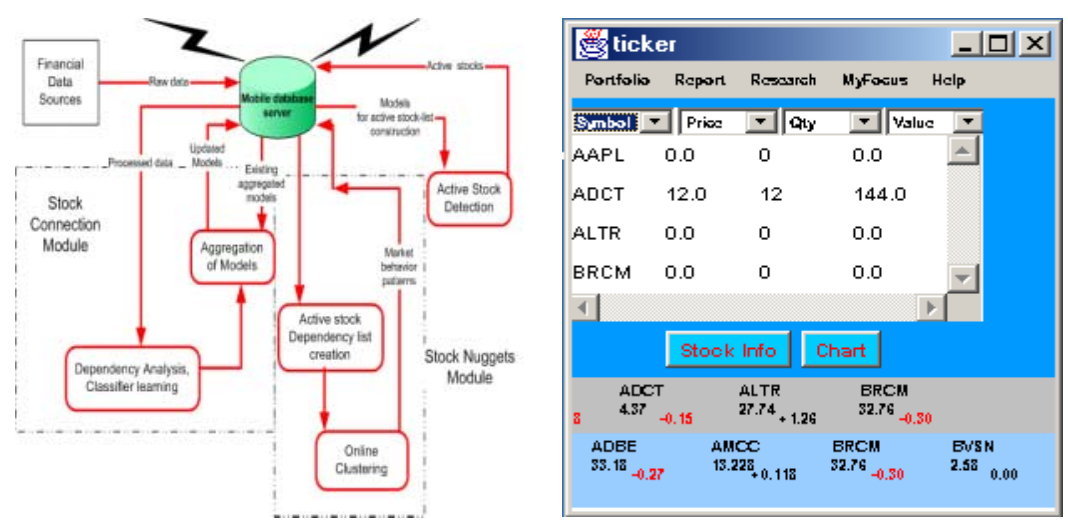

Fig. 1. (Left) The architecture of the MobiMine Server. (Right) The main interface of MobiMine. The bottom-most ticker shows the WatchList; the ticker right above the WatchList shows the stocks in the portfolio

\section{The MobiMine System}

This section presents an overview of the MobiMine, a PDA-based application for managing stock portfolios and monitoring the continuous stream of stock market data. The overview presented in this section covers the different modules of the MobiMine; not all of them make use of the random matrix-based techniques discussed so far in this paper. However, a general description is necessary to cast the current contribution in the context of a real application environment.

\subsection{An Overview of the System}

The MobiMine is a client-server application. The clients (Figure 2), running on mobile devices like hand-held PDAs and cell-phones, monitor a stream of financial data coming through the MobiMine server (Figure 1(Top)). The system is designed for currently available low-bandwidth wireless connections between the client and the server. In addition to different standard portfolio management operations, the MobiMine server and client apply several advanced data mining techniques in order to offer the user a variety of different tools for monitoring the stock market at any time from any where. Figure 1(Bottom) shows the main user interface of the MobiMine.

The main functionalities of the MobiMine are listed in the following:

1. Portfolio Management and Stock Tickers: Standard book-keeping operations on stock portfolios including stock tickers to keep an eye on the performance of the stocks in the portfolio.

2. FocusArea: Stock market data is often overwhelming. It is very difficult to keep track of all the developments in the market. Even for a full-time 


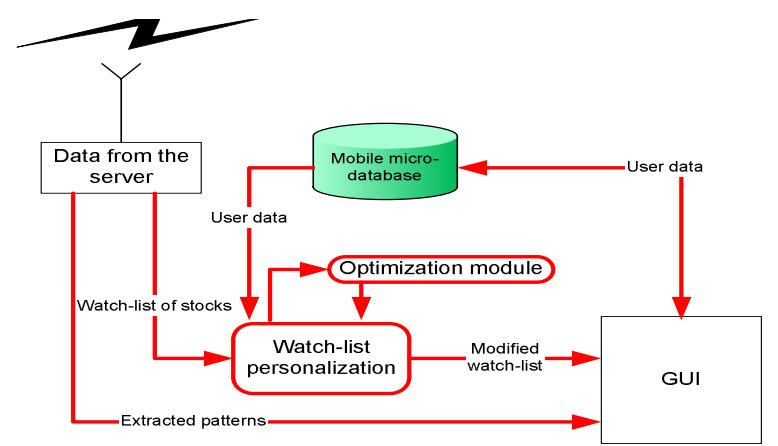

Fig. 2. The architecture of the MobiMine Client

professional following the developments all the time is challenging. It is undoubtedly more difficult for a mobile user who is likely to be busy with other things. The MobiMine system offers a unique way to monitor changes in the market data by selecting a subset of the events that is more "interesting" to the user. This is called the FocusArea of the user. It is a time varying feature and it is currently designed to support the following functionalities:

(a) WatchList: The system applies different measures to assign a score to every stock under observation. The score is an indication of the "interesting-ness" of the stock. A relatively higher score corresponds to a more interesting stock. A selected bunch of "interesting" stocks goes through a personalization module in the client device before it is presented to the user in the form of a WatchList.

(b) Context Module: This module offers a collection of different services for better understanding of the time-critical dynamics of the market. The main interesting components are,

i. StockConnection Module: This module allows the user to graphically visualize the "influence" of the currently "active" stocks on the user's portfolio. This module detects the highly active stocks in the market and presents the causal relationship between these and the stocks in user's portfolio, if any. The objective is to give the user a high level qualitative idea about the possible influence on the portfolio stocks by the emerging market dynamics.

ii. StockNuggets Module: The MobiMine Server continuously processes a data stream defined by a large number of stock features (fundamentals, technical features, evaluation of a large number of wellknown portfolio managers). This module applies online clustering algorithms on the active stocks and the stocks that are usually influenced by them (excluding the stocks in the user's portfolio) in order to identify similarly behaving stocks in a specific sector. 
The StockConnection module tries to detect the effect of the market activity on user's portfolio. On the other hand, the StockNuggets module offers an advanced stock-screener-like service that is restricted to only time-critical emerging behavior of stocks.

(c) Reporting Module: This module supports a multi-media based reporting system. It can be invoked from all the interfaces of the system. It allows the user to watch different visualization modules and record audio clips. The interface can also invoke the e-mail system for enclosing the audio clips and reports.

A detailed description of this system can be found elsewhere [2]. The following section discusses the unique philosophical differences between the MobiMine and traditional systems for mining stock data.

\subsection{MobiMine: What It Is Not}

A large body of work exists that addresses different aspects of stock forecasting $[3,4,5,6,7,8]$ and selection $[9,10]$ problem. The MobiMine is fundamentally different from the existing systems for stock forecasting and selection. First of all, it is different on the basis of philosophical point of view. In a traditional stock selection or portfolio management system the user initiates the session. User outlines some preferences and then the system looks for a set of stocks that satisfy the constraints and maximizes some objective function (e.g. maximizing return, minimizing risk). The MobiMine does not do that. Instead it initiates an action, triggered by some activities in the market. The goal is to draw user's attention to possibly time-critical information. For example, if the Intel stock is under-priced but its long time outlook looks very good then a good stock selection system is likely to detect Intel as a good buy. However, the MobiMine is unlikely to pick Intel in the WatchList unless Intel stock happens to be highly active in the market and it fits with user's personal style of investment. The Context detection module is also unlikely to show Intel in its radar screen unless Intel happens to be highly influenced by some of the highly active stocks in the market. This difference in the design objective is mainly based on our belief that mobile data mining systems are likely to be appropriate only for time-critical data. If the data is not changing right now, probably you can wait and you do not need to keep an eye on the stock price while you are having a lunch with your colleagues.

This paper focuses on the correlation-based dependency detection aspect of the used in the StockConnection module. The following section initiates the discussion.

\section{Data Filtering and Random Matrices}

Detecting dependencies among stocks is an important task of MobiMine for identifying the focus area of the user. Correlation analysis of time series data is a 
common technique for detecting statistical dependencies among them. However, doing it online is a challenging problem since correlation must be computed from incrementally collected noisy data. At any given instant, the MobiMine can compute the correlation matrix among a set of stocks. However, the correlation may be completely misleading introduced by many factors like noise and small number of observations.

Accurate estimation of the correlation requires proper filtering of the correlation matrix. This paper considers an approach that removes the "noise" by considering the eigen values of the covariance matrix computed from the collected data. The noisy eigen-states are removed by exploiting properties of the eigen-distribution of random matrices. The eigenvalues of the covariance matrix can then be used, in conjunction with random matrix theory, to identify and filter out the noisy eigenstates.

In this section, we will first present a brief review of the theory of random matrices. We will then present the EDS, that works incrementally by observing one block of data at a time.

\subsection{Introduction to Random Matrices}

A random matrix $X$ is a matrix whose elements are random variables with given probability laws. The theory of random matrices deals with the statistical properties of the eigenvalues of such matrices.

In this paper, we would be interested in the distribution of the eigenvalues of the sample covariance matrix obtained from a random matrix $X$. Let $X$ be an $m \times n$ matrix whose entries $X_{i j}, i=1, \ldots, m, j=1, \ldots, n$ are i.i.d. random variables. Furthermore, let us assume that $X_{11}$ has zero mean and unit variance. Consider the $n \times n$ sample covariance matrix $Y_{n}^{(m)}=\frac{1}{m} X X^{\prime}$. Let $\lambda_{n 1}^{(m)} \leq \lambda_{n 2}^{(m)} \leq$ $\cdots \leq \lambda_{n n}^{(m)}$ be the eigenvalues of $Y_{n}^{(m)}$. Let $F_{n}^{(m)}(x)=\left(\sum_{i=1}^{n} U\left(x-\lambda_{n i}^{(m)}\right)\right) / n$, be the empirical cumulative distribution function (c.d.f.) of the eigenvalues $\left\{\lambda_{n i}^{(m)}\right\}_{1 \leq i \leq n}$, where $U(x)$ is the unit step function. We will consider asymptotics such that in the limit as $N \rightarrow \infty$, we have $m(N) \rightarrow \infty, n(N) \rightarrow \infty$, and $\frac{m(N)}{n(N)} \rightarrow Q$, where $Q \geq 1$.

Under these assumptions, it can be shown that [11] the empirical c.d.f. $F_{n}^{(m)}(x)$ converges in probability to a continuous distribution function $F_{Q}(x)$ for every $x$, whose probability density function (p.d.f.) is given by

$$
f_{Q}(x)= \begin{cases}\frac{Q \sqrt{\left(x-\lambda_{\min }\right)\left(\lambda_{\max }-x\right)}}{2 \pi x} & \lambda_{\min }<x<\lambda_{\max } \\ 0 & \text { otherwise }\end{cases}
$$

where $\lambda_{\min }=(1-1 / \sqrt{Q})^{2}$ and $\lambda_{\max }=(1+1 / \sqrt{Q})^{2}$.

\subsection{EDS Approach for Online Filtering}

Consider a data stream mining problem that observes a series of data blocks $X_{1}$, $X_{2}, \cdots X_{s}$, where $X_{t}$ is an $m_{t} \times n$ dimensional matrix observed at time $t$ (i.e., $m_{t}$ 
observations are made at time $t$ ). If the data has zero-mean, the sample covariance $\operatorname{Cov}_{t}$ based on data blocks $X_{1}, X_{2}, \ldots, X_{t}$ can be computed in a recursive fashion as follows [12]:

$$
\operatorname{Cov}_{t}=\frac{\sum_{j=1}^{t-1} m_{j}}{\sum_{j=1}^{t} m_{j}}\left[\operatorname{Cov}_{t-1}+\frac{m_{t}}{\sum_{j=1}^{t-1} m_{j}} \hat{\Sigma}_{t}\right]
$$

where $\hat{\Sigma}_{i}=\left(X_{i}^{\prime} X_{i}\right) / m_{i}$ is the sample covariance matrix computed from only the data block $X_{i}$.

In order to exploit the results from random matrix theory, we will first center and then normalize the raw data, so that it has zero mean and unit variance. This type of normalization is sometimes called Z-normalization, which simply involves subtracting the column mean and dividing by the corresponding standard deviation. Since the sample mean and variance may be different in different data blocks (in general, we do not know the true mean and variance of the underlying distribution), Equation 2 must be suitably modified. In the following, we provide the important steps involved in updating the covariance matrix incrementally. Detailed derivations can be found in [12].

Let $\mu_{t}, \sigma_{t}$ be the local mean and standard deviation row vectors, respectively, for the data block $X_{t}$ and $\overline{\mu_{t}}, \overline{\sigma_{t}}$ be the aggregate mean and standard deviation, respectively, based on the aggregation of $X_{1}, X_{2}, \cdots, X_{t}$. Let $\bar{X}_{r}, \hat{X}_{r}$ denote the local centered and local Z-normalized data, respectively, obtained from data block $X_{r}(1 \leq r \leq t)$ using $\mu_{r}$ and $\sigma_{r}$. Moreover, at time $t$, let $\bar{X}_{r, t}, \hat{X}_{r, t}$ denote the actual centered and actual Z-normalized data obtained from data block $X_{r}$ using the $\overline{\mu_{t}}$ and $\overline{\sigma_{t}}$. In particular, $\hat{X}_{r, t,[i, j]}=\bar{X}_{r, t,[i, j]} / \overline{\sigma_{t,[j]}}=\left(X_{r, t,[i, j]}-\right.$ $\left.\overline{\mu_{t,[j]}}\right) / \overline{\sigma_{t,[j]}}$, where $i, j$ denote row and column indices. Note that the aggregate mean $\overline{\mu_{t}}$ can be updated incrementally as follows: $\overline{\mu_{t}}=\left(\sum_{r=1}^{t} \mu_{r} m_{r}\right) / \sum_{r=1}^{t} m_{r}$ $=\left(\overline{\mu_{t-1}} \sum_{r=1}^{t-1} m_{r}+\mu_{t} m_{t}\right) / \sum_{r=1}^{t} m_{r}$. Let us define $Z_{t}$ to be the covariance matrix of the aggregation of centered (or zero mean) data $\overline{X_{1, t}}, \overline{X_{2, t}}, \ldots, \bar{X}_{t, t}$, and $z_{t}$ be the local covariance matrix of the current block $\bar{X}_{t}$. Note that

$$
\overline{\sigma_{t,[j]}}=\sqrt{Z_{t,[j, j]}}, \text { and } \operatorname{Cov}_{t,[i, j])}=\frac{Z_{t,[i, j]}}{\overline{\sigma_{t,[i]}} \times \overline{\sigma_{t,[j]}}}, \quad 1 \leq i, j \leq n,
$$

where $\operatorname{Cov}_{t}$ is the covariance matrix of the aggregated Z-normalized data $\hat{X}_{1, t}, \ldots, \hat{X}_{t, t}$. Therefore, the Z-normalization problem is reduced to that of incrementally updating the covariance matrix $Z_{t}$ on the centered data. Define $\overline{\Delta_{t}}=\left(\overline{\mu_{t}}-\overline{\mu_{t-1}}\right)$ and $\Delta_{t}=\left(\overline{\mu_{t}}-\mu_{t}\right)$. It is then easy to show that [12]

$$
\begin{aligned}
\bar{X}_{r, t}^{\prime} \bar{X}_{r, t}-\bar{X}_{r, t-1}^{\prime} \bar{X}_{r, t-1} & =m_{r} \bar{\Delta}_{t}^{\prime} \bar{\Delta}_{t}, \quad \bar{X}_{t, t}^{\prime} \bar{X}_{t, t}-\left(\bar{X}_{t}\right)^{\prime}\left(\bar{X}_{t}\right)=m_{t} \Delta_{t}^{\prime} \Delta_{t}, \quad \text { and } \\
Z_{t} & =Z_{t-1}+\bar{\Delta}_{t}^{\prime} \bar{\Delta}_{t} \sum_{r=1}^{t-1} m_{r}+\bar{X}_{t}^{\prime} \bar{X}_{t}+m_{t} \Delta_{t}^{\prime} \Delta_{t}
\end{aligned}
$$

The above discussion shows that the covariance matrix can be incrementally updated, which can then be used to compute eigenvectors. An online algorithm 

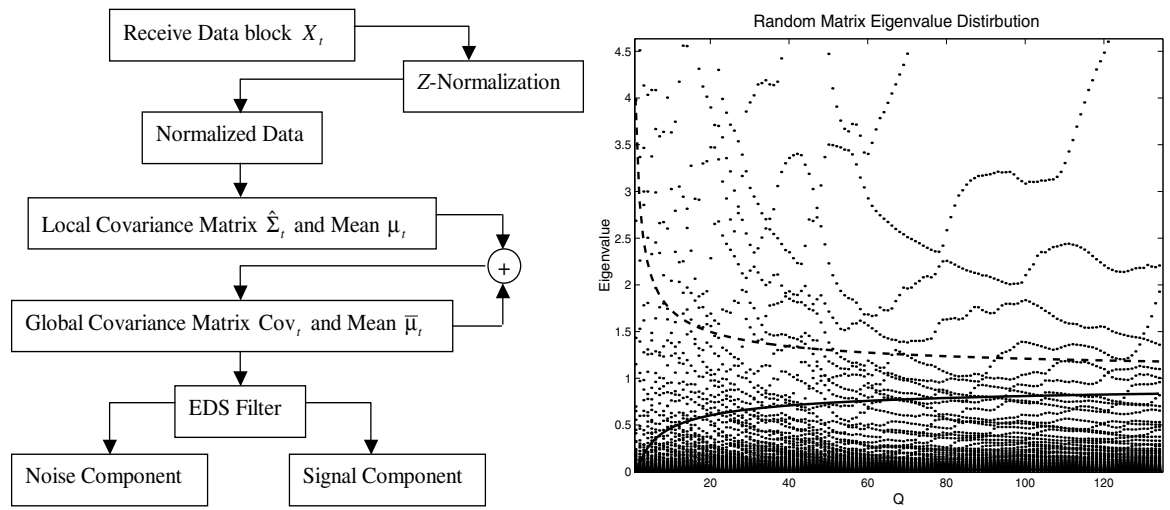

Fig. 3. (Left) The flow chart of the proposed EDS approach for mining data streams. (Right) The distribution of the eigen values, $\lambda_{\max }$ and $\lambda_{\min }$ with increasing Q. The graph is generated using the financial data set

can directly compute the eigenvectors of this matrix. However, this simplistic approach does not work well in practice due to two main problems: (a) data may be inherently noisy and (b) the number of observations $\left(m_{i}\right)$ made at a given time may be small. Both of these possibilities may produce misleading covariance matrix estimates, resulting in spurious eigen-states. It is important that we filter out the noisy eigenstates and extract only those states that belong to the eigenspace representing the underlying information.

In this paper, we assume that the observed data is stationary and consists of actual information corrupted by random noise. The proposed technique decomposes the covariance matrix into two components: (1) the noise part and (2) the information part by simply comparing the eigenspace of the covariance matrix of observed data with that of a randomly generated matrix. In other words, we compare the distribution of the empirically observed eigenvalues with the theoretically known eigenvalue distribution of random matrices given by Equation 1 . All the eigenvalues that fall inside the interval $\left[\lambda_{\min }, \lambda_{\max }\right]$ correspond to noisy eigenstates. Following are some of the main steps at any time $t$ in the EDS approach:

1. Based on the current estimate of the covariance matrix $\mathrm{Cov}_{t}$, compute the eigenvalues $\lambda_{t, 1} \leq \cdots \leq \lambda_{t, n}$.

2. Identify the noisy-eigenstates $\lambda_{t, i} \leq \lambda_{t, i+1} \cdots \leq \lambda_{t, j}$ such that $\lambda_{t, i} \geq \lambda_{\text {min }}$ and $\lambda_{t, j} \leq \lambda_{\max }$. Let $\Lambda_{t, n}=\operatorname{diag}\left\{\lambda_{t, i}, \ldots, \lambda_{t, j}\right\}$, be the diagonal matrix with all the noisy eigenvalues. Similarly, let $\Lambda_{t, s}=\operatorname{diag}\left\{\lambda_{t, 1}, \ldots, \lambda_{t, i-1}, \lambda_{t, j+1}, \ldots\right.$, $\left.\lambda_{t, n}\right\}$, be the diagonal matrix with all the non-random eigenvalues.

Let $A_{t, n}$ and $A_{t, s}$ be the matrices whose columns are eigenvectors corresponding to the eigenvalues in $\Lambda_{t, n}$ and $\Lambda_{t, s}$, respectively and $A_{t}=\left[A_{t, s} \mid A_{t, n}\right]$. Then we can decompose $\operatorname{Cov}_{t}=\operatorname{Cov}_{t, s}+\operatorname{Cov}_{t, n}$, where $\operatorname{Cov}_{t, s}=A_{t, s} \Lambda_{t, s} A_{t, s}^{\prime}$ is the signal 
part of the covariance matrix and $\operatorname{Cov}_{t, n}=A_{t, n} \Lambda_{t, n} A_{t, n}^{\prime}$ is the noise part of the covariance matrix. At any given time step, the signal part of the covariance matrix produces the useful non-random eigenstates and they should be used for data mining applications. Note that it suffices to compute only the eigenvalues (and eigenvectors) that fall outside the interval $\left[\lambda_{\min }, \lambda_{\max }\right]$, corresponding to the signal eigenstates. This allows computation of $\operatorname{Cov}_{t, s}$ and hence $\operatorname{Cov}_{t, n}$. The following section documents the performance of the EDS algorithm for detecting dependencies among Nasdaq 100 stocks.

\section{Mobile Financial Data Stream Mining Using EDS}

In order to study the properties of the proposed EDS technique in a systematic manner, we performed controlled experiments with Nasdaq 100 financial timeseries data streams. The EDS is used to generate a "filtered" correlation matrix that detects the dependencies among different stocks. This section reports the experimental results.

The experiments reported here consider 99 companies from Nasdaq 100. We sample data every five minutes and each block of data is comprised of $m_{i}=99$ rows. At any given moment the user is interested in the underlying dependencies observed from the current and previously observed data blocks.

First let us study the effect of the EDS-based filtering on the eigen-states produced by this financial time-series data. Figure 3 (Right) shows the distribution of eigenvalues from the covariance matrix $\left(\mathrm{Cov}_{t}\right)$ for different values of $t$. It also shows the theoretical lower and upper bounds $\left(\lambda_{\max }\right.$ and $\left.\lambda_{\min }\right)$ at different time steps (i.e. increasing $Q$ ). The eigen-states falling outside these bounds are considered for constructing the "signal" or "information" part of the covariance matrix. The figure shows that initially a relatively large number of eigen states are identified as noisy. As time progresses and more data blocks arrive, the noise regime shrinks. It is also interesting to note that the EDS algorithm includes the lower end of the spectrum in the signal part. This is philosophically different from the traditional wisdom of considering only those eigen states with large eigen values.

In order to evaluate the online performance of the EDS algorithm we compare the eigen-space captured by the EDS at any given instant with respect to the "true" eigen-space defined by the underlying data distribution. Although data streams are conceptually infinite in size, the experiments documented in this section report results over a finite period of time. So we can benchmark the performance of the EDS with respect to the "true" eigen-space defined by the eigen vectors computed from the entire data set (all the data blocks) collected from the stream during the chosen period of observation. We first report the evolution of the signal-part of the covariance matrix as a function of time and compare that with the "true" covariance matrix generated from the entire data set. We report two different ways to compute this difference:

1. RMSE: The root mean square error (RMSE) is computed between the covariance matrices generated by the online EDS and the entire data set. 

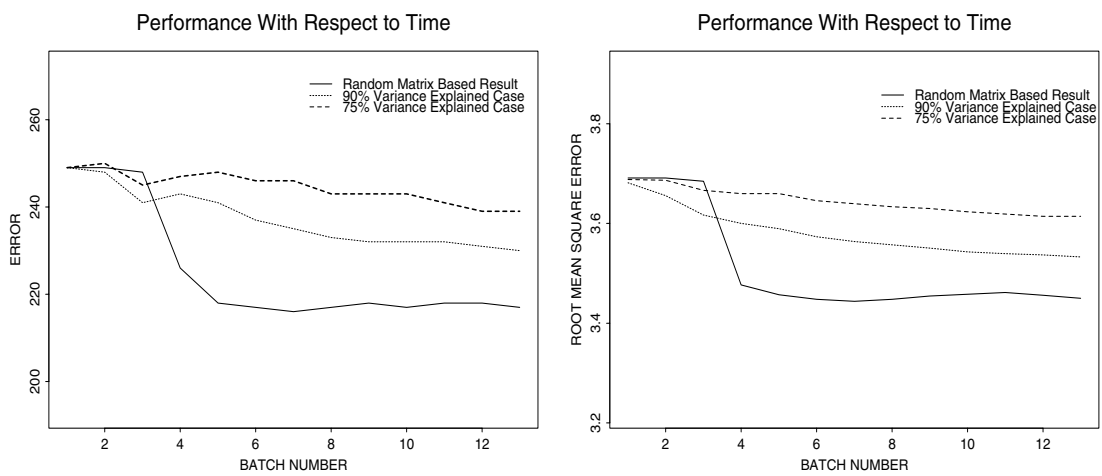

Fig. 4. The relative performance (thresholded error count in left and RMS error on right) of the EDS algorithm and the traditional approach using eigen vectors capturing $90 \%$ and $75 \%$ of the total variance. Different batch numbers correspond to different time steps

2. Thresholded Error: This measure first computes the difference between the estimated and true covariance matrices. If the $(i, j)$-th entry of the difference matrix is greater than some user given threshold $\theta$ then the value is set to 1 otherwise 0 . The total number of 1 's in this matrix is the observed thresholded error count.

Figure 4 compares the performance of our EDS algorithm with that of a traditional method that simply designates as signal, all the eigen-states that account for, respectively, $90 \%$ and $75 \%$ of the total variance. The latter corresponds a regular principal component analysis (PCA) that uses enough components to explain, respectively, $90 \%$ and $75 \%$ of the total variation in data. It shows the thresholded error count for each method, as a function of time (batch number). It is apparent that the EDS algorithm quickly outperforms the traditional method.

In order to evaluate the effect of filtering performed by the EDS algorithm, we picked a representative pair of stocks and compared the covariance between them, computed from the EDS filtered data and compare it with that using the raw data directly. We plot the absolute error between the estimated covariance value at each time step and the final covariance value.

Figure 5 depicts the comparison for the covariance between two stocks Dell (DELL) and Amazon (AMZN). It is apparent that filtering using the EDS algorithm results in a smaller error overall.

\section{Conclusions and Future Work}

Although mobile computing devices are becoming more accessible and computationally powerful, their usage is still restricted to simple operations like web 


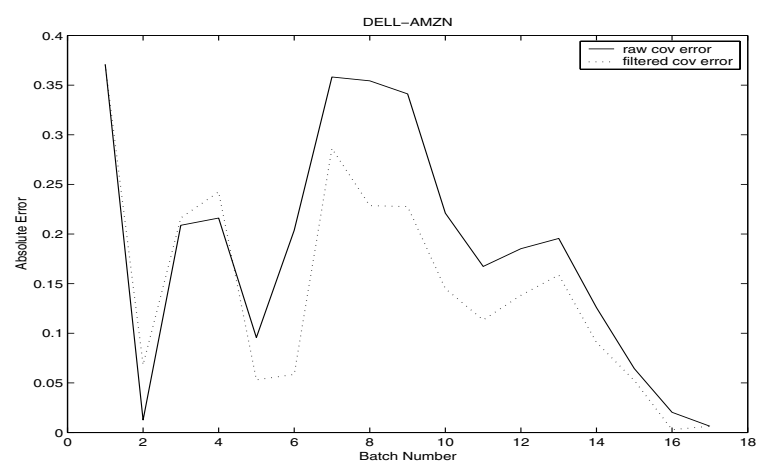

Fig. 5. Comparison of error in correlation estimates with and without EDS filtering for correlation between Dell and Amazon

surfing, checking emails, maintaining personal schedules, and taking notes. The limited battery power, restricted visual display area, and the low-bandwidth communication channel are hindering more sophisticated desktop-style applications. However, the ubiquitous aspect of these devices makes them very attractive for many time-critical applications. We need a new breed of applications for time-critical domains that can live with these resource restrictions.

This paper described one such application that performs online mining of financial data. It offered the EDS approach for extracting useful noise-free eigen states from data streams. It shows that the EDS approach is better than the traditional wisdom of selecting the top-ranking eigenvectors guided by some usergiven threshold. The EDS allows us to extract the eigenstates that correspond to non-random information that are likely to be useful from a data mining perspective. Indeed, any data mining technique, exploratory or otherwise, can be applied on the EDS filtered data.

The EDS approach works by comparing the empirically observed eigen distribution with the known distribution of random matrices. The theoretically known values of upper and lower limits of the spectrum are used to identify the boundary between noisy and signal eigen-states.

Another feature of our EDS approach is illustrated in Figure 3. As seen from the graph, the limits $\lambda_{\max }$ and $\lambda_{\min }$ both converge to 1 as the ratio $Q$ tends to infinity (see also equation 1 ). In a data stream mining scenario, the number of features $n$ is fixed, whereas the number of total number of observations $m$ increases as each block of data is received. Hence, $Q$ increases with time, which results in a smaller interval $\left[\lambda_{\max }, \lambda_{\min }\right]$ for the noisy eigenstates. This means that, as we observe more and more data, the EDS algorithm would potentially designate more eigenstates as signal. This is also intuitively satisfying, since most of the noise would get "averaged-out" as we observe more data.

In many real-world applications, the data stream is usually quasi-stationary. In other words, the data can be considered to be stationary over short periods 
of time but the statistical behavior of the data changes - either gradually over time or due to some underlying event that triggers an abrupt change. These situations can be easily accommodated in the EDS framework. For example, one can use a fixed finite window of past observations to update the covariance matrix. Alternatively, an exponential weighting can be applied to past data blocks, thereby relying more on the recent data. Abrupt changes in the data distribution can be detected by monitoring the change in the covariance matrix or the subspace spanned by the noisy eigenstates, using an appropriate metric. Any significant deviation with respect to the past history would be an indication of an abrupt change. The EDS filter can be reset in such circumstances. We plan to pursue some of these ideas in a future publication.

\section{Acknowledgments}

The authors acknowledge supports from the United States National Science Foundation CAREER award IIS-0093353 and NASA (NRA) NAS2-37143. The authors would like to thank Li Ding for his contribution to related work and Qi Wang for performing some of the experiments.

\section{References}

1. M. L. Mehta. Random Matrices. Academic Press, London, 2 edition, 1991. 251

2. H. Kargupta, H. Park, S. Pittie, L. Liu, D. Kushraj, and K. Sarkar. MobiMine: Monitoring the stock market from a PDA. ACM SIGKDD Explorations, 3:37-47, 2001. 254

3. A. Azoff. Neural Network Time Series Forecasting of Financial Markets. Wiley, New York, 1994. 254

4. N. Baba and M. Kozaki. An intelligent forecasting system of stock price using neural networks. In Proceedings IJCNN, Baltimore, Maryland, pages 652-657, Los Alamitos, 1992. IEEE Press. 254

5. S. Cheng. A neural network approach for forecasting and analyzing the pricevolume relationship in the taiwan stock market. Master's thesis, National JowTung University, Taiwan, R.O.C, 1994. 254

6. R. Kuo, L. Lee, and C. Lee. Intelligent stock market forecasting system through artificial neural networks and fuzzy delphi. In Proceedings of World Congress on Neural Networks, pages 345-350, San Deigo, 1996. INNS Press. 254

7. C. Lee. Intelligent stock market forecasting system through artificial neural networks and fuzzy delphi. Master's thesis, Kaohsiung Polytechnic Institute, Taiwan, R.O.C, 1996. 254

8. J. Zirilli. Financial Prediction Using Neural Networks. International Thomson Computer Press, 1997. 254

9. J. Campbell, A. Lo, and A. MacKinley. The Econometrics of Financial Markets. Princeton University Press, USA, 1997. 254

10. G. Jang, F. Lsi, and T. Parng. Intelligent stock trading decision support system using dual adaptive-structure neural networks. Journal of Information Science Engineering, 9:271-297, 1993. 254 
11. D. Jonsson. Some limit theorems for the eigenvalues of a sample covariance matrix. Journal of Multivariate Analysis, 12:1-38, 1982. 255

12. H. Kargupta, S. Ghosh, L. Ding, and K. Sivakumar. Random matrices and online principal component analysis from data streams. Submitted to The Eighth ACM SIGKDD International Conference on Knowledge Discovery and Data Mining, 2002. 256 